\title{
Gas type identification at constant concentration level by system identification technique
}

\begin{abstract}
The purpose of this paper is to explore the ability of the system identification technique in detecting a specific gas at a constant concentration level by a single sensor. Metal oxide semiconductor (MOS) gas sensor is used due to its wide range of applicability in gas monitoring systems. Using the Batch computer method, 115 outputs were recorded with sampling time of $0.57 \mathrm{Sec}$ at a concentration level of 2600 (ppm) for Methanol, Ethanol, and Betanol gases. Applying the estimated model, each gas was successfully distinguished.
\end{abstract}

Keyword: Gas; Sensors; System identification; Metal oxide semiconductor; MOS 